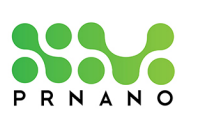

Prec. Nanomed. 2020;3(1):483-486

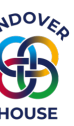

\title{
11 Years of CLINAM, the European Foundation for Clinical Nanomedicine
}

\author{
Beat Löffler, MD h.c. MA \\ CEO, European Foundation for Clinical Nanomedicine
}

Submitted: February 3, 2020

Accepted: February 7, 2020

Published: February 11, 2020

The basic unit of all living organisms, the cell, is constructed out of a multitude of smaller structures, the organelles. These consist of biomolecules that interact with each other and which unite mechanical and biochemical functions on the nanoscale. These molecular nanomachines build the basis of all living organisms and it is the mission of the European Foundation for Clinical Nanomedicine (CLINAM) to advance medicine to the benefit of individuals and society through the application of nanoscience, nanotechnology, and targeted medicine in healthcare.

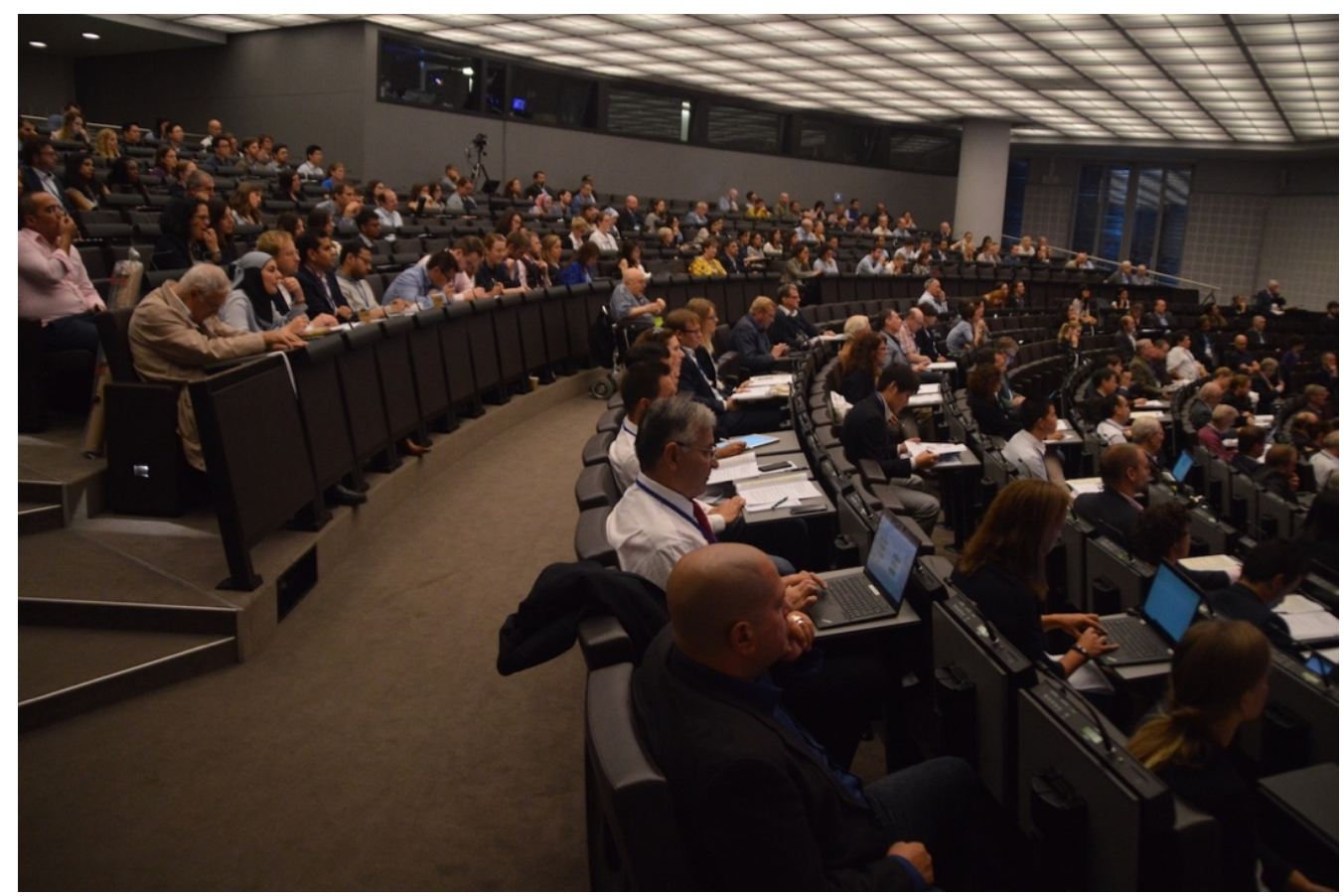

Figure 1. Participants at the plenary session, 2018, Basel.

CLINAM is a nonprofit organization based in Basel, Switzerland (www.clinam.org), that has been founded by Beat Löffler and Patrick Hunziker in $2007^{*}$. The organization, utilizing its worldwide network of stakeholders, hosts the annual European and Global Summit for Clinical Nanomedicine in the fields of nanomedicine, targeted drug delivery, precision medicine, and other related fields. These summits relate to the main elements of cutting-edge medicine and the inclusion of the implications of nanomedicine for patients and mankind. On 4/26/2009, CLINAM founded the International Society for Nanomedicine (ISNM) with the participation of scientists and national organizations from Japan.

*Dr. Beat Löffler, MD h.c., MA is the CEO of CLINAM and is responsible for the management and the dissemination projects of CLINAM, for the shaping of Summit Programs, and for organizing all conferences and exhibitions. He runs his company, Löffler \& Associates to translate visions to reality by developing the right strategies since 1994. His company also organizes other conferences, such as in materials technology, and energy supply development.

Prof. Dr. Patrick Hunziker, MD is a clinician, cardiologist, and co-leader of the Intensive Care Unit of the University Hospital Basel. He is the leader of the CLINAM Lab and president of the International Society for Nanomedicine. He has been researching nanomedicine as a clinician for 15 years. He is involved in several large international research projects. 
Korea, USA, Canada, Europe, South America, India, Australia, Africa, Australia, and India and is led by another member country every 2 years. Every 2 years, ISNM organizes a summer school, which brings together experienced researchers and students. Another important element of CLINAM is the European Society for Nanomedicine (www.esnam.org), which has more than 1000 members today. CLINAM also supports Precision Nanomedicine ${ }^{\#}$, the official journal of the foundation.

CLINAM pursues a comprehensive catalogue of projects, that recognises the major impact of nanoscience on medicine, and the rapid advancement it means for medical applications including clinical nanomedicine and targeted medicine.

The organization, utilizing its worldwide network of stakeholders, hosts the European and Global Summit for Clinical Nanomedicine in the fields of nanomedicine, targeted drug delivery, precision medicine, patient-centric medicine and other related fields (after this year's May 17-20, Basel meeting, the next is scheduled for September 2022). At these meetings, keynote addresses delivered by Nobel laureates are followed by 10-minute scientific highlights providing ample time for discussions during session breaks, lunch, and evening cultural programs.

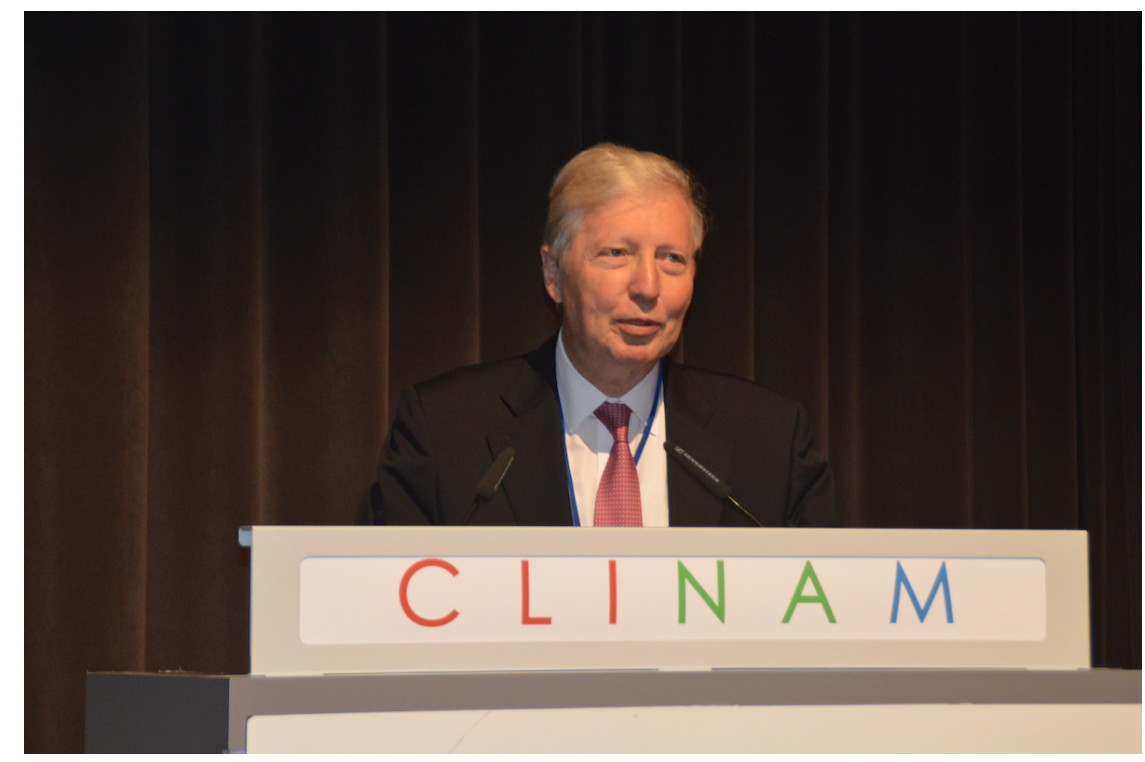

Figure 2. Prof. Dr. Jules Hoffmann (Institute of Molecular and Cellular Biology, CNRS), opening speaker at CLINAM 2018 as one of the three Nobel laureates (Prof. Dr. Ada Yonath and Prof. Dr. Harald zur Hausen) speaking at the meeting.

Up to date, CLINAM has organized 11 European \& Global Summits for Clinical Nanomedicine. Aiming at prevention, diagnosis, and therapy through nanomedicine, the foundation enhances clinically focused research, increases interactions and improves the information flow between clinicians, researchers, the public, and all stakeholders in the field of nanomedicine.

These summits relate to the main elements of cutting-edge medicine and the inclusion of the implications of nanomedicine for patients and mankind. These meetings proved to be the place to be because discussions and interactions have resulted in successful collaborations, gather novel ideas, and learn about innovative methodologies and technologies as well as new projects, including numerous Europe-wide and international efforts. Five years ago, regulatory authorities of all continents also chose CLINAM as a neutral nonprofit platform for their International Pharmaceutical Regulators Programme meeting (IPRP).

\footnotetext{
\# Precision Nanomedicine (www.prnano.com) is a platinum open access, international, peer-reviewed, onlineonly journal that promotes all progressive and rational aspects of nanomedicine. The journal is published by Andover House, Inc., a not-for-profit scientific publisher, owned and led by scientists and professionals in New England, USA, a traditional hub for science and medicine (www.andoverhouse.org). Precision Nanomedicine is indexed and/or abstracted in Crossref, in the Directory of Open Access Journals, and archived in Portico. The journal is a member of the Committee in Publication Ethics (COPE) and the San Francisco Declaration on Research Assessment.
} 


\begin{tabular}{lccccc} 
Year & \multicolumn{1}{c}{ Participants } & Countries & Talks & Posters & Liaisons \\
2007 & The FOUNDATION registered and started on April 23 & \\
2008 & 158 & 24 & 83 & 18 & 4 \\
2009 & 250 & 30 & 109 & 24 & 8 \\
2010 & 280 & 33 & 102 & 29 & 7 \\
2011 & 335 & 31 & 108 & 76 & 9 \\
2012 & 351 & 34 & 105 & 80 & 3 \\
2013 & 450 & 36 & 150 & 120 & 4 \\
2014 & 470 & 41 & 161 & 111 & 6 \\
2015 & 497 & 42 & 172 & 115 & 4 \\
2016 & 511 & 39 & 191 & 121 & 3 \\
2017 & 531 & 42 & 195 & 125 & 4 \\
2018 & 489 & 43 & 199 & 129 & 2 \\
\hline
\end{tabular}

Figure 3. Growth of CLINAM Summits (https://www.clinam.org/past-conferences.html).

Throughout the years, CLINAM summits have become an international melting pot, a globally unique networking platform, and a unique scientific event that brings together all stakeholders in nanomedicine targeted medicine and precision medicine. The ensuing vivid discussions motivate every member of the community.

A major benefit of these summits is that pioneers both in Nanoscience in Health, as well as many researchers, opinion leaders, students, startups, established companies, and regulators all participate. Face-to-face meetings with regulators allow to create trust, and many stakeholders approach representatives of regulatory authorities to receive guidelines for their early-stage development. This opportunity may considerably accelerate the approval process of new medicines and procedures. CLINAM leaders and its Scientific Board understand that nanomedicine is constantly evolving and keep offering fresh and innovative technologies for personalized medicine.

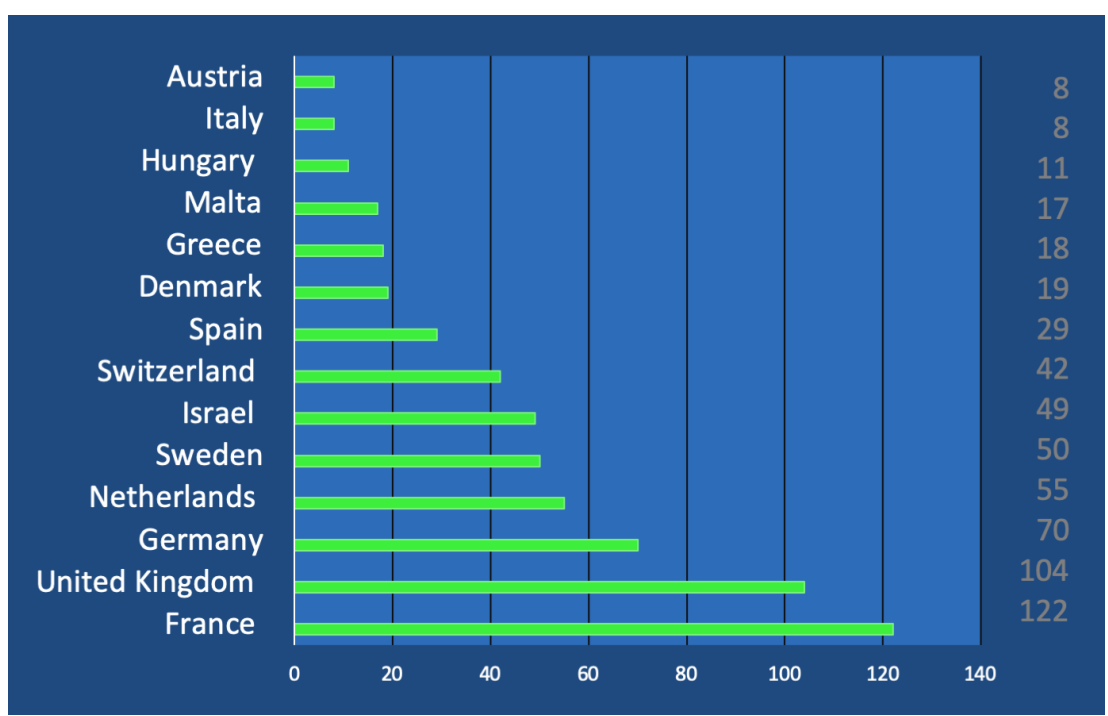

Figure 4. 620 Nano-Drug Projects in the European Pipeline in Research, Preclinical, Phase 1,2,3, Approved, and Marketed. 12 other countries share another 20 projects.

The next Summit titled Clinical Nanomedicine and the Impact of Digitalization and Artificial Intelligence for Precision Medicine - The Technologies for Diagnosis and \& Therapy in Personalized Medicine will be held in Basel, Switzerland, Oct 25-28, 2020. In its $12^{\text {th }}$ Conference, the Foundation emphasizes the role of digitalization and artificial intelligence The topic demonstrates the competence 
of the foundation to keep up with the signs of time. At this meeting, CLINAM again brings together opinion leaders, pioneers, experts, and a new generation of researchers to debate and interact on nanomedicine, genetics, digitalization, artificial intelligence (AI), and other novel technologies. Based on recent groundbreaking achievements of nanomedicine and related fields, the meeting will concentrate on how to shape the path to personalized medicine.

Many companies such as Ionis, The Medicines Company, Novo-Nordisk, Caribou Biosciences, Novartis, Roche, Alnylam Pharmaceuticals, CureVac, Moderna Therapeutics, Bayer, and others will debate about new modalities approaching on previously undruggable biological targets, such as intracellular ones. This interaction between researchers and the industry is of great value for both sides. For members of startups, these contacts often lead to cooperation and support.

Digitalization and AI systems are needed to store, handle, and understand those immense data sets arising in medicine. High-performance computing with massive computational power will be necessary to gain a more detailed understanding not only of the generic aspects of diseases but how they may present themselves in an individual. AI can and will be used in comprehensive analysis and in advanced simulations in virtual patients, to predict the most effective and least dangerous treatment strategy that can be applied to each person individually in a cost-effective manner.

New tools, materials, and strategies must be developed to improve the effectiveness of drugs and procedures. Progressive understanding of the genome and the human immune system in this field enables the translation of basic science to innovative, effective, and affordable medical applications. Particular attention will be given to potential benefits, but also to the inherent risks and pitfalls of artificial intelligence and machine learning.

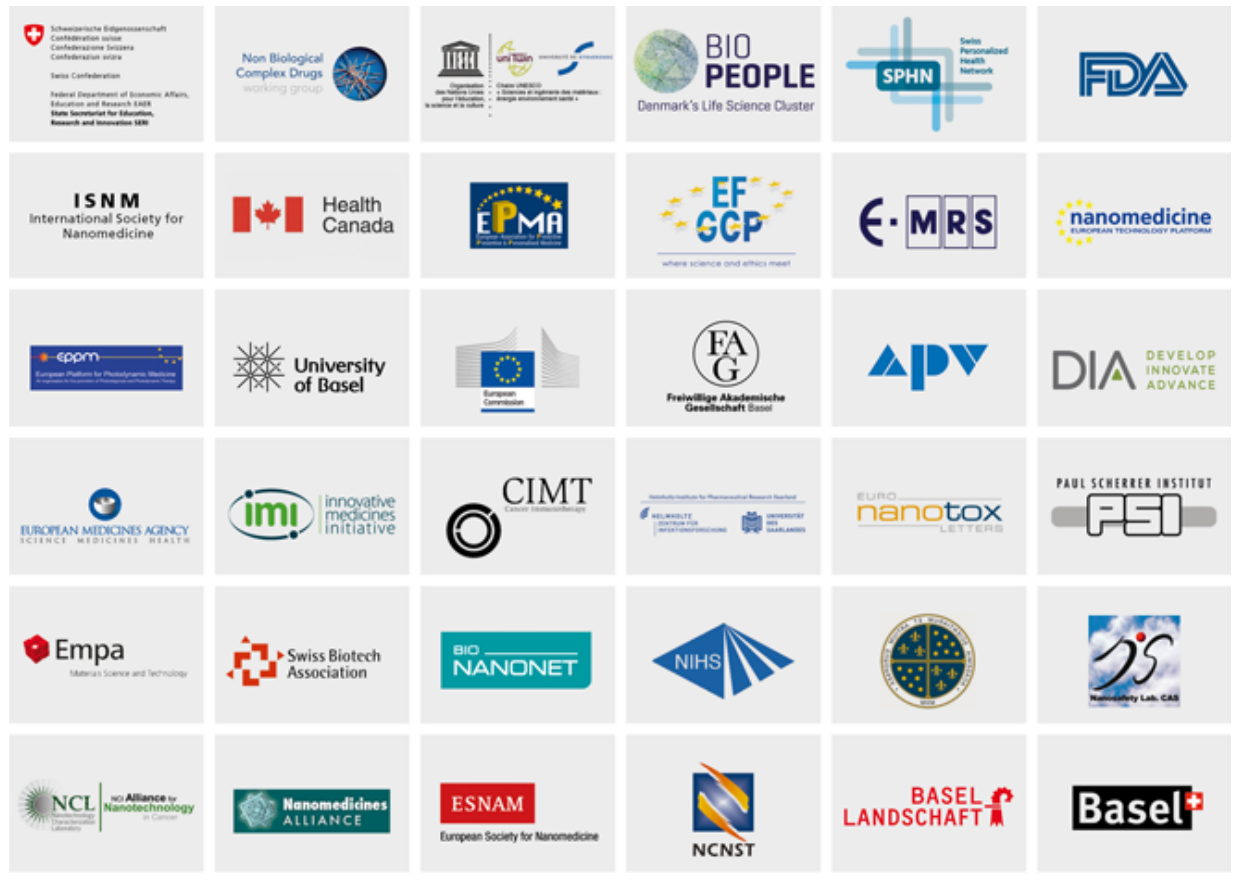

Figure 5. Summit under the auspices of the Swiss Confederation with 36 NPO supporters.

The board of the CLINAM Foundation has been cooperating with many nonprofit organizations that are willing and able to bring skilled expertise to applications of nanotechnology in health. Members of the CLINAM family share their interdisciplinary experience in an interactive manner that widens mutual understanding and serves as a great motivation for all. The goal of all participants is to continue to use this neutral European and Global platform for nanomedicine development in convergence with many related technologies.

\section{Come, learn, participate in the discussions, and become part of the CLINAM Family!}

\section{www.clinam.org}

Quote this article as Löffler B, 11 Years of CLINAM, the European Foundation for Clinical

Nanomedicine, Prec. Nanomed. 2020;3(1):483-486, https://doi.org/10.33218/001c.11897 